\section{THU0703-HPR A SANCTUARY FROM EVERYDAY LIFE: A QUALITATIVE STUDY OF THE EXPERIENCE OF IN- PATIENT MULTIDISCIPLINARY REHABILITATION, FOR PATIENTS WITH RHEUMATIC DISEASES}

Maria Stauner ${ }^{1}$, Jette Primdahl $l^{2,3,4} .{ }^{1}$ Clinical institute, Odense, Denmark; ${ }^{2}$ University of Southern Denmark, Department of Regional Health Resarch, Odense, Denmark; ${ }^{3}$ Hospital of Southern Jutland, Aabenraa, Denmark; ${ }^{4}$ King Christian Xth Hospital for Rheumatic Diseases, Gråsten, Denmark

Background: In Denmark, patients with rheumatic diseases can receive inpatient multidisciplinary rehabilitation at a hospital for rheumatic diseases or at rehabilitation centers.

Quantitative studies indicate that it is difficult to detect a general and lasting effect of 1-4 weeks of inpatient multidisciplinary rehabilitation, but few studies have explored patients' experience of in-patient multidisciplinary rheumatology rehabilitation.

Objectives: To explore how patients, experience the process and personal impact of an inpatient rehabilitation stay.

Methods: An exploratory qualitative phenomenological-hermeneutic study was planned. Adult rheumatic patients admitted for a two-week inpatient rehabilitation stay were invited to participate. Individual semi-structured interviews were conducted in the patients' home shortly after discharge or at the ward one of the last days of admission. The interviews were audio recorded and transcribed verbatim. The analysis was inspired by Paul Ricour's interpretative philosophy (1).

Results: Fifteen interviews were conducted, 11(73\%) female, age 28-89. The analysis derived a core theme," $A$ sanctuary", reflecting that the patients experienced to have sufficient time and mental resources to provide self-care. In addition, the analysis derived five subthemes: 1) "Being seen, heard and acknowledged as an equal and whole person". To feel acknowledged was vital for the patients' experience of quality and benefit. 2) "Professional care and compassion", which were considered by the patients as the most fundamental contextual factors to facilitate their selfcare. 3) "Social relations and interactions between patients", reflecting the patients experience of feeling recognized by other patients and to experience common understanding. 4) "An individually planned rehabilitation stay, but challenges regarding shared decision making". The patients felt the rehabilitation was individually planned, but with room for improvement in relation to awareness of shared decision-making. 5) "Rehabilitation as a personal process but problems with transferability to everyday life". The patients experienced the rehabilitation stay as a part of a personal rehabilitation process, but expressed concerns about whether they were able to transfer new learning and habits to everyday life.

Conclusion: Patients with rheumatic diseases experience in-patient rehabilitation as a sanctuary, with rehabilitation at three levels; through multidisciplinary rehabilitation interventions at the hospital; through recognition from the multidisciplinary staff and through recognition, social relationships and interactions with fellow patients. There is a need for improved coordination of rehabilitation across primary and secondary health care, in order to ease transferal to the patients' everyday life.

\section{REFERENCES:}

[1] Dreyer PS, Pedersen BD. Distanciation in Ricoeur's theory of interpretation: narrations in a study of life experiences of living with chronic illness and home mechanical ventilation. Nurs Inq. 2009;16(1):64-73.

Disclosure of Interests: None declared

DOI: 10.1136/annrheumdis-2019-eular.1528

\section{THU0704-HPR PATIENT ACTIVATION AND ADHERENCE TO BIOLOGICAL THERAPY AND TARGETED SYNTHETIC DMARD: PRELIMINARY RESULTS:}

A Sendra-García ${ }^{1,2}$, P Llopis-Salvia ${ }^{3}$, Marta Hermenegildo-Caudevilla ${ }^{4}$, Marta Aguilar-Zamora ${ }^{5}$, L Montolio-Chiva ${ }^{5}$, Ana Victoria Orenes Vera ${ }^{5}$, I VázquezGómez $^{5}$, À Martínez-Ferrer ${ }^{5}$, D Ybáñez-García ${ }^{5}$, Elia Valls-Pascual ${ }^{5}$, V NúñezMonje ${ }^{5}$, I Torner-Hernández ${ }^{5}$, Mónica Climente-Marti ${ }^{3}$, Juanjo J Alegre-Sancho ${ }^{5}$. ${ }^{1}$ Universitly Hospital Doctor Peset, Rheumatology and Pharmacy Department, València, Spain; ${ }^{2}$ Fisabio, València, Spain; ${ }^{3}$ Dr. Peset University Hospital, Pharmacy Department, València, Spain; ${ }^{4}$ Dr. Peset University Hospital, Pharmacy Department, Valencia, Spain; ${ }^{5}$ Dr. Peset University Hospital, Rheumatology, Valencia, Spain

Background: Medication non-adherence has been associated with treatment failure in chronic inflammatory conditions. A positive relationship between patient activation and adherence to treatment and between activation and improved clinical outcomes has been shown for chronic conditions.

Objectives: To measure adherence to biological therapies and targeted synthetic DMARD (tsDMARD), and their relationship with the Patient Activation Measure (PAM) and with patient and therapy related factors, for chronic inflammatory arthropathies.

Methods: Cross-sectional observational descriptive study in a general tertiary university hospital.

Patients on treatment with the same biological drug (subcutaneous; phase 1) or tsDMARD (oral; phase 2) for $\geq 6$ months were included in order of arrival and those with mental disability, which prevented understanding of the study, were excluded.

Demographic variables (sex, age, living environment, educational level), diagnosis and treatment were collected. Adherence was measured using the Simplified Medication Adherence Questionnaire (SMAQ) for biological therapies, the Compliance Questionnaire Rheumatology (CQR-19) for tsDMARD, and the medication possession ratio (MPR). Patients were considered adherent if MPR $\geq 80 \%$ and CQR-19 $\geq 80 \%$ or adherent SMAQ. To measure activation, PAM questionnaire, was used. Patients were classified as activated or not activated.

Statistical analysis: relationship between adherence to treatment and PAM was analyzed using chi-square, considering significance level $p$ $<0,05$. Statistical analysis was performed with spss $v 17.0$.

Results: A total of 58 patients (57\% women) were included. Mean age was 54 years $(95 \% \mathrm{Cl}$ : 50 to 57$), 86 \%$ lived in urban areas, $40 \%$ had completed elementary education, 31\% high-school and $24 \%$ university studies.

Distribution by diagnosis: rheumatoid arthritis (81\%), ankylosing spondylitis $(14 \%)$ and psoriatic arthritis $(5 \%)$. Distribution by treatment was: baricitinib $(31 \%)$, tofacitinib $(18 \%)$, adalimumab $(16 \%)$, tocilizumab $(14 \%)$, etanercept $(12 \%)$, secukinumab $(7 \%)$ and golimumab $(3 \%)$. Median time of disease duration was 9 years (IQR 13) and time on treatment with the drug was 8 months (IQR 20), with significant differences between groups (27 (biological therapy) vs 7 months (tsDMARD)).

The proportion of adherent patients was $43 \%$, being higher among tsDMARD $(57 \%)$ vs biological treated ones $(30 \%)$, with significant differences.

$72 \%$ of patients were activated, being more activated biological $(80 \%)$ than tsDMARD $(67 \%)$ treated patients.

A higher adherent proportion of patients was found among the activated patients (48\%) compared to the non-activated ones $(27 \%)$ in all measures, even though the differences were not statistically significant.

Conclusion: Patients treated with biological therapies and tsDMARD have a high degree of activation for the self-management of their disease and its treatment. Adherence to treatment may be influenced by route of administration, type of drug (oral/tsDMARD $57 \%$ vs. subcutaneous/biological $30 \%$ ) and time on treatment (longer time on treatment, lesser adherence).

The greater proportion of adherence found among patients with a higher degree of activation could indicate a positive relationship between activation and adherence, so analyzing and promoting patient activation seems important in order to improve adherence to drugs.

Disclosure of Interests: None declared

DOI: 10.1136/annrheumdis-2019-eular.6956

\section{THU0705-HPR PELVIC FLOOR AND SEXUAL DYSFUNCTION IN WOMEN WITH SJOGREN SYNDROME}

Alime Buyuk Gonen 1 , S. Yaprak Cetin 1 , Ayse Ayan ${ }^{2} .{ }^{1}$ Akdeniz University, Deartment of Physiotherapy, ANTALYA/TURKEY, Turkey, ${ }^{2}$ Antalya Education and Research Hospital, Department of Rheumatology, ANTALYATTURKEY, Turkey

Background: Sjogren syndrome (SS) is a systemic autoimmune disease causing secretory gland dysfunction. This leads to dryness of the mainly salivary and lacrimal glands such as the mouth, eyes, nose, larynx, and vagina. The disease overwhelmingly affects middle aged women. These women have symptoms of decreased lacrimal and salivary gland function which usually precede involvement of other exocrine glands such as the upper airway, the gastrointestinal tract and the external genitalia (dyspareunia). Although genital tract symptoms are common in patients with sjogren, such as vulvar and vaginal dryness, dyspareunia, and pruritus, their pelvic floor health and functions have been rarely evaluated.

Objectives: The aim of study was investigation of pelvic floor and sexual dysfunction in patients with SS.

Methods: 23 patients with SS with an average age of $49.00 \pm 8.71$ were included in the study. Pelvic floor dysfunction of patients with SS were evaluated by the Pelvic Floor Impact Questtionare-7 (PFIQ-7) and Pelvic 